\title{
Design of Metallic Phase Diagram Determinator Based on Electromagnetic Heating Technology
}

\author{
Hu Jun-ping ${ }^{1,2, a}$, Ren Yun ${ }^{1}$ and Xie Peng-tao ${ }^{1}$ \\ ${ }^{1}$ School of Chemical and Materials of Handan College, Handan, 056005, China \\ ${ }^{2}$ Chemistry and Chemical engineering Experiment Teaching Center of Handan College, \\ Handan 056005, China \\ a71917640@qq.com
}

Keywords: Electromagnetic heating, Metallic phase diagram, Temperature control effect.

\begin{abstract}
A novel kind of metallic phase diagram determinator has been developed in order to deal with the problem of original determinator big thermal inertia and bad temperature control. Which is used by the electromagnetic heating technology. The determinator has many functions such as temperature setting, data collection, heating, cooling control by fan and regular alarm etc. The experimental results agreed well with the theoretical value through experimentally tested by using improved tube. It was shown that the novel determinator has high precision of temperature control, saving and environmentally, safe.
\end{abstract}

\section{Introduction}

Two component of metallic phase diagram drawing is one of the basic experiment of physical chemistry, university mainly measurement system with the method of thermal analysis in the process of heating or cooling, the system temperature with the change of time metal sample phase transition temperature is derived, and drawing phase diagram [1]. And the whole experiment is the key to precisely control the heating temperature [2] of the sample. Physical chemistry experiment in colleges and universities mainly use Nanking Sang Li electronic technology co., Ltd. Production of metal phase diagram experiment instrument, heating mode is used in heating wire heating technology, the electrical energy is converted to heat energy in heat transfer way for sample after heated. The technology is influenced by the environment, high power consumption, heating speed is slow, especially in the heating stopped, but because of the heating wire waste heat, sample temperature will continue to rise, it is difficult to control, the serious influence the accuracy of experimental results. If improper operation, easy to cause system overheat, sealing material violent boiling, even threatened the personal safety of users [3]. Based on this, this study by designing a kind of the electromagnetic induction heating technology of metallic phase diagram experiment instrument makes every effort to simplify the experimental operation, to improve the temperature control effect, improve the accuracy of determination results.

\section{Instrument Design of Electromagnetic Heating Metal Phase Diagram Tester}

Traditional metal phase diagram using the technology of the heating wire heating, heating device is the use of heated material itself and the conductivity of poor conductor features to heating, heating for the contact. In the metal phase diagram experiment, the thermal hysteresis and thermal inertia big shortcoming, not accurate temperature control, lead to the result of the experiment error [4]. Replace the heating mode, control the generation of waste heat becomes the key to design experimental apparatus of metallic phase diagram. As shown in Fig. 1 electromagnetic induction heating technology is a new type of heating technology, which uses high frequency electric heating principle, will have a high frequency alternating current into high frequency current, magnetic field, magnetic field lines in the magnetic field through the insulation board effect on heated objects, the lines of magnetic force was cut, the tiny vortex flow, make the object itself fever quickly, so as to achieve the 
purpose of heating. The overall energy conversion form from the process of electricity - magnetic energy-energy-heat, electromagnetic induction coil itself does not produce heat, basically can effectively reduce the generation of waste heat, realize a sample system inside and outside temperature, real-time accurate temperature control. At the same time has safe and reliable, high efficiency and energy saving, long life advantages.

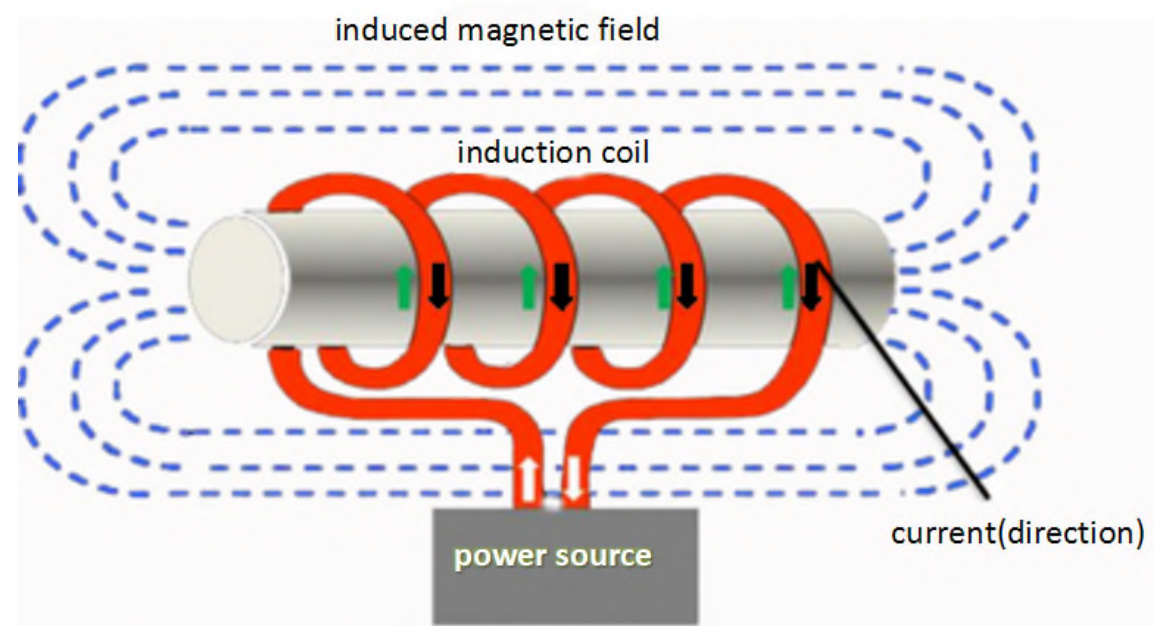

Fig. 1 The principle diagram of the electromagnetic induction heating technology.

\section{Principle of Electromagnetic Heating Metal Phase Diagram Tester}

New electromagnetic heating the working principle of experimental apparatus of metallic phase diagram as shown in Fig. 2, there are mainly integrated two major part is the host and electromagnetic induction heating furnace. Host and temperature controlled by integration, electromagnetic induction heating, fan, time, voice controller and so on six parts, temperature setting, data acquisition, heating, cooling fan speed control, timing alarm, etc. The most outstanding characteristic is under the single chip microcomputer control of temperature and battery induction heating power real-time control function. First of all, the temperature sensor is composed of armored thermocouple nickel chrome, copper, insert in the middle of sample tube, heating temperature real-time monitoring samples, and feedback the temperature to the temperature controller module. Single-chip microcomputer control system with temperature controller communicate with each other, real-time understand the relationship between samples of set temperature and induction temperature, and issuing commands to the controller, electromagnetic induction heating control of electromagnetic induction heating furnace heating condition, and according to the set temperature and temperature difference, timely adjust the magnetic intensity of electromagnetic induction, the nonlinear change of eddy current sensor, changing the heating power of the heating furnace and improve temperature control accuracy [5].

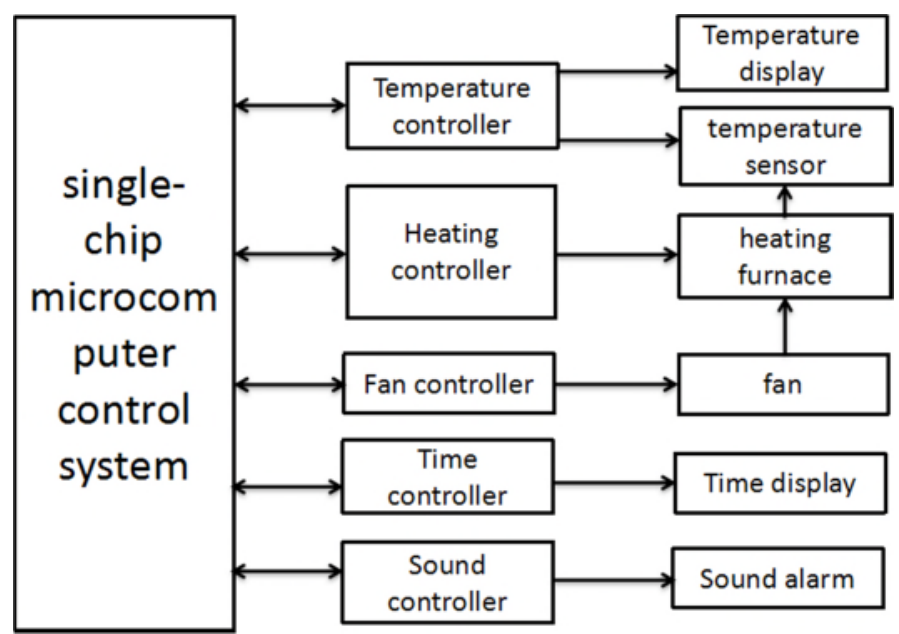

Fig. 2 Principle of new type of electromagnetic heating metal phase diagram tester. 


\section{Integrated System Design}

According to the working principle of new type of electromagnetic heating metal phase diagram tester requirements, the system overall design as shown in Fig. 3: points as the main circuit and control circuit part. Main circuit for power transformation, mainly including the main resonance circuit and EMI filter circuit, circuit diagram in this paper, escapes in concrete structure. Control circuit is responsible for the control of the whole system and the realization of the intelligent, is the key to realize intensive and intelligent, mainly accomplished by hardware and software of the control system. This design system for internal memory, the interrupt source, I/O ports and so on various aspects of the request is not high, choose ordinary MCU AT89S52 devices of Atmel company.AD sampling chip need the number of channels is not more than eight road, accuracy, using the integrated multiplex switch ADC08038 chip can meet the requirements. In use process, microcontroller through the 4 way of input signal, the input current, output current, the analysis of the sample temperature, set temperature input, judge whether the load of the heating furnace is provided in conform to the requirements, the output the corresponding power, and according to the thermocouple temperature and set temperature difference, in a timely manner to adjust the size of the output power. Prompted to set up, after the completion of the heating time, automatically countdown state, output voice prompt signals after zero. Throughout the process, in order to stable temperature control system, set a cooling fan under the heating furnace, by adjusting speed cooling fan control signal. Display output signal consists of light-emitting diodes (leds), according to the sample temperature, the periodic time [6].

Software part of the total process as shown in Fig. 4, initialized at the beginning of the system, set up the sample of heating temperature, because there is no heat, electromagnetic heating technology in this experiment set temperature in $40 \sim 50{ }^{\circ} \mathrm{C}$ higher than that of the sample melting point position. SCM in according to the size of the input and output current, and the relationship between the both direct scanning heating furnace in the load, and the validity of the load, to avoid too much electricity damage electronic components. Thermocouple temperature detected sample by AD acquisition card transfer to the single-chip microcomputer control system, all input signal detection system and normal, through introspection. Such as fault occurs, the system stop output current, on the instrument panel screen display the fault type, help users inspection equipment problems in a timely manner. Self-checking by instrument began formal output current to heat the samples, sample temperature real time acquisition system, and compared with the set temperature, is below the set temperature, continue to heating system. When is higher than the set temperature, stop heating, and began to cool, at the same time start the timer function, according to the need to set the countdown time, and in the corresponding interval to remind operator to write down temperature, until the end of the experiment.

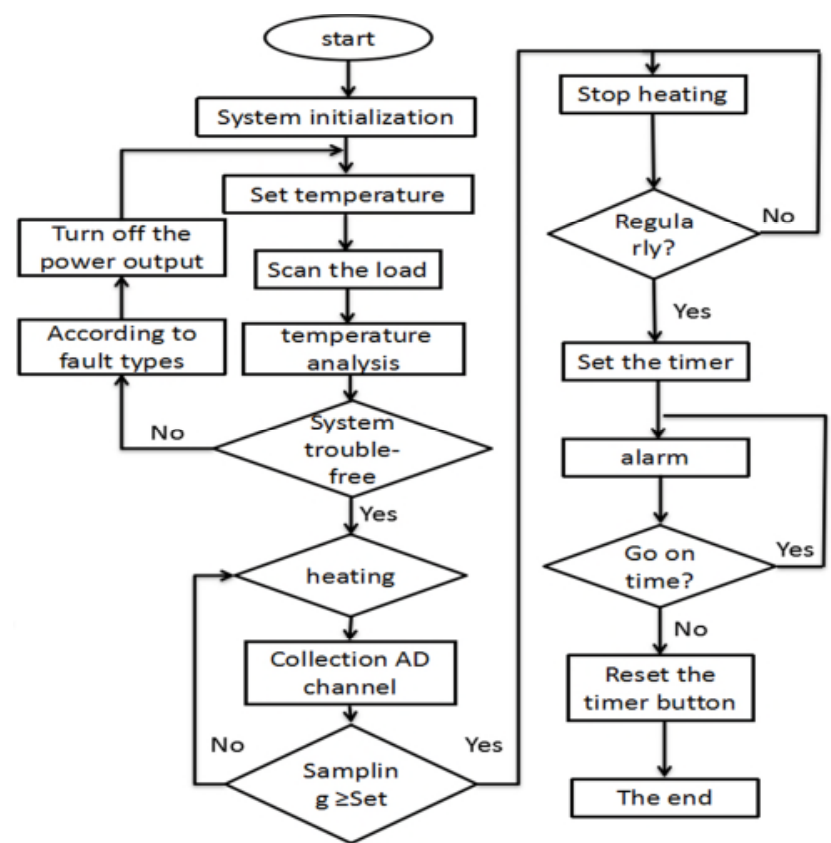

Fig. 3 Part of the total flow chart of system software. 


\section{Metal Phase Diagram to Determine the Design of the Test Tube}

Electromagnetic heating for heating body must be to magnetic materials [7]. As shown in Fig. 4, in the original metal phase diagram to determine the outside of the glass tube, nested carbon steel casing, good magnetic conductivity due to the carbon steel and high resistance and can satisfy the requirement of the electromagnetic heating receptor. When put improved test tubes in the heating furnace, under the effect of electromagnetic heating, the carbon steel casing began to fever, again in the form of heat transfer in heating tube samples, armored thermocouple in-depth internal sample, and sample temperature detecting real-time feedback, reach the set temperature, the host to stop output current, heating stops. Carbon steel casing and cooling to the sample. Improved electromagnetic heating tube and metal phase diagram tester, significantly reduce the influence of waste heat to the system, and can satisfy experiment requirement.

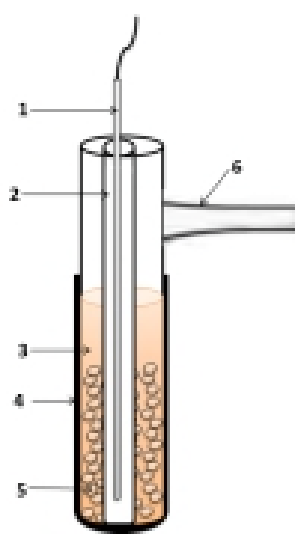

1 - temperature sensor; 2, in vitro (insert sensor); 3 - liquid paraffin (sample sealing liquid); 4 - carbon steel casing; 5 - metal sample; 6 - tube entrance;

Fig. 4 determination of metallic phase diagram tube structure diagram.

\section{Application}

Metal lead and metal tin prepared lead level 0, 20, 38.1, 60, 80, 100, six samples of each $100 \mathrm{~g}$, placed in a metal phase diagram in a test tube, insert electromagnetism furnace respectively, the specific requirements for operation experiment step reference guide book, record sample cooling temperature and the corresponding time, in order to draw cooling curve and phase diagram, as shown in Fig. 5, lead tin alloy cooling curve and binary phase diagram and phase diagram theory close, error is smaller. Results show that the new types of electromagnetic heating metal phase diagram tester obviously enhance the temperature control of the system, improve the accuracy and the experimental results to optimize. In addition, also has safe and reliable, energy conservation, environmental protection, long service life, the promotion of significance and application value.

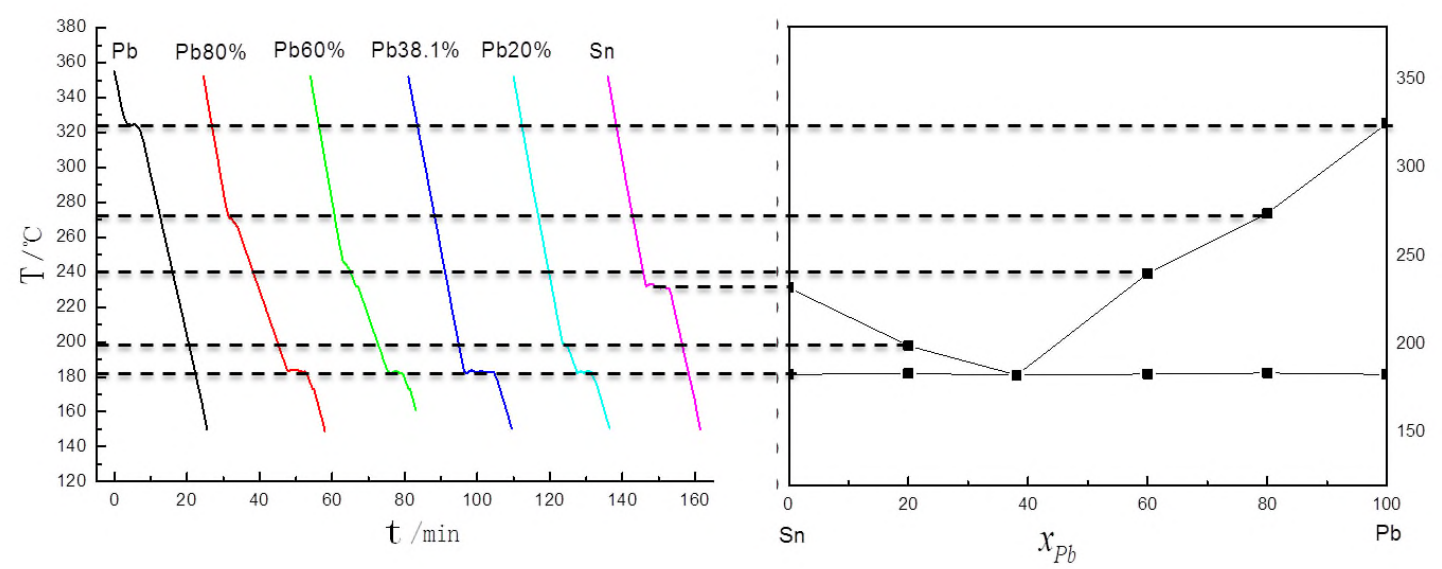

Fig. 5 Lead tin alloy cooling curve and binary phase diagram. 


\section{References}

[1] J. H. Zhuang, Physical chemistry experiment, (Third edition). Beijing: higher education press, 2005.

[2] Z. H. Huang, H. Zhao, L. J. Lv, Thermal analysis technique and its application, J. Therm. Process. 7 (2010) 19-22.

[3] F. G. Chen, J. Chen, A practical new type of metal phase diagram experimental stove, J. Lab. Res. Explor. 1 (2001) 84-86.

[4] W. Z. Luo, S. John and H. Y. Zhao, Magnetic field distribution in the different heating element under the conditions of electromagnetic induction heating research, J. Therm. Process. 22 (2013) 95-98.

[5] Y. J. Zhao, The research and application of electromagnetic induction heating technology. Xi 'an: northwestern polytechnic university, 2007.

[6] G. M. Song and Y. T. Wang, Electromagnetic induction heating system circuit design, J. Wei Fang College J. 4 (2010) 34-36.

[7] M. F. Wu, X. G. Wang, H. K. Hu, Two component alloy phase diagram experiment improvement research, J. Lab. Sci. 5 (2010) 88-90. 